Wilfrid Laurier University

Scholars Commons @ Laurier

Physics and Computer Science Faculty

Publications

Physics and Computer Science

2004

\title{
Entanglement Dynamics in Chaotic Systems
}

Shohini Ghose

Wilfrid Laurier University, sghose@wlu.ca

Barry C. Sanders

University of Alberta

Follow this and additional works at: https://scholars.wlu.ca/phys_faculty

\section{Recommended Citation}

Ghose, Shohini and Sanders, Barry C., "Entanglement Dynamics in Chaotic Systems" (2004). Physics and Computer Science Faculty Publications. 62.

https://scholars.wlu.ca/phys_faculty/62

This Article is brought to you for free and open access by the Physics and Computer Science at Scholars Commons @ Laurier. It has been accepted for inclusion in Physics and Computer Science Faculty Publications by an authorized administrator of Scholars Commons @ Laurier. For more information, please contact scholarscommons@wlu.ca. 


\title{
Entanglement dynamics in chaotic systems
}

\author{
Shohini Ghose ${ }^{1,2}$ and Barry C. Sanders ${ }^{1,3}$ \\ ${ }^{1}$ Institute for Quantum Information Science, University of Calgary, Alberta, Canada T2N 1N4 \\ ${ }^{2}$ Department of Physics and Astronomy, University of New Mexico, Albuquerque, New Mexico 87131, USA \\ ${ }^{3}$ Centre for Quantum Computer Technology, Macquarie University, Sydney, New South Wales, Australia \\ (Received 22 July 2004; revised manuscript received 9 September 2004; published 16 December 2004)
}

\begin{abstract}
We study quantum chaos for systems with more than one degree of freedom, for which we present an analysis of the dynamics of entanglement. Our analysis explains the main features of entanglement dynamics and identifies entanglement-based signatures of quantum chaos. We discuss entanglement dynamics for a feasible experiment involving an atom in a magneto-optical trap and compare the results with entanglement dynamics for the well-studied quantum kicked top.
\end{abstract}

DOI: 10.1103/PhysRevA.70.062315

PACS number(s): 03.67.Mn, 05.45.Mt, 03.65.Ud, 39.25.+k

\section{INTRODUCTION}

Theories of chaos and of quantum mechanics juxtapose in the discipline of "quantum chaos" (QC), which has attracted significant theoretical and experimental [1] efforts. Recently the advent of quantum information [2] has highlighted the role of entanglement as a resource, and stimulated theoretical studies of entanglement in QC systems [3-8]. Whereas maximal entanglement can be created in many systems without chaos, QC systems may generate entanglement at a faster rate. Here we present a general analysis of entanglement dynamics for unitarily evolving QC systems, and apply this analysis to a system of significant experimental interest: an atom in a magneto-optical lattice (AMOL), which we show is a feasible experimental system to observe and test entanglement dynamics and to rapidly enhance entanglement production for certain initial states. The theoretical methods and results are general, which we demonstrate by application to the well-studied quantum kicked top (QKT) [6,9-11]. We also identify the initial entanglement rate as a signature of quantum chaos.

Entanglement features of QC systems can be subtle. Recent theoretical studies have revealed that entanglement may be enhanced as the chaoticity parameter is increased [3,6]. Other studies indicate that increased chaos can lead to a saturation of the rate of entanglement generation [5]. Entanglement generation in a bipartite system depends on both the coupling strength between the two systems as well as the degree of chaos as shown by Jacquod [8]. Hence, the rate of entanglement generation between two subsystems can vary depending on whether the total bipartite system is globally strongly chaotic or whether the two subsystems are each individually strongly chaotic but weakly coupled. Whereas our focus is on the former case, which can yield a rapid increase of entanglement, our multiqubit analysis of the entanglement dynamics of a single kicked top can be used to understand the saturation in entanglement obtained for coupled kicked tops.

We focus our attention on the evolution of an AMOL $[12,13]$ under realistic experimental conditions and show that entanglement arises between the atomic spin and motional degrees of freedom. The ability to tomographically reconstruct the reduced density matrix of the atomic spin state [14] makes it possible to study the evolution of this entanglement experimentally. We analyze the dynamics of entanglement (via the entropy of the spin density matrix) and characterize the global dynamics by the size of the chaoticity parameter and local dynamics by whether the initial state is supported primarily by regular or chaotic eigenstates of the Hamiltonian. AMOL experiments would allow the first empirical studies of entanglement evolution in a QC system.

An AMOL provides an attractive framework for studying entanglement evolution in a QC system with and without coupling to an environment. This is because, in addition to having more than one degree of freedom and the ability to tomographically reconstruct states, decoherence can be controlled by detuning the laser relative to the atomic resonance frequency. Decoherence is negligible for the far offresonance AMOL so coupling to the environment can be ignored (unitary dynamics), and entanglement between the spin and motional subsystems of the overall chaotic system can be explored. By tuning the laser frequency close to atomic resonance, coupling to the environment is increased and the resulting effects of entanglement with the environment can be observed. Here we are concerned with the former case of the far off-resonance magneto-optical lattice for which coupling to the environment can be neglected and entanglement is enhanced. We show that the AMOL can exhibit generic features of entanglement dynamics, for example quasiperiodicity for a state initially localized in a regular regime and a rapid increase of entanglement with no subsequent quasiperiodicity in a chaotic regime.

\section{ANALYSIS OF ENTANGLEMENT DYNAMICS}

Generic features of entanglement in QC systems can be understood by examining the spectral properties of the evolution operator $U(t)$ on the system Hilbert space $\mathcal{H}=$ $\otimes_{i=1}^{N} \mathcal{H}^{(i)}$ with $\mathcal{H}^{(i)}$ the Hilbert space of dimension $d_{i}$ for the $i$ th subsystem. We consider two common categories of unitary evolution: (i) $U(t)=\exp (-i H t / \hbar)$ for a timeindependent Hamiltonian $H$ and time $t$, and (ii) $U(t=n \tau)$ $=F^{n}$ with $F$ a Floquet operator $F=T \exp \left[-i / \hbar \int_{0}^{\tau} H(t) d t\right]$. The evolution operator can be expressed as 


$$
U(t)=\sum_{j}^{d} \exp \left(-i \omega_{j} t\right)\left|\phi_{j}\right\rangle\left\langle\phi_{j}\right|
$$

for $\left\{\left|\phi_{j}\right\rangle\right\}$ a time-independent orthonormal basis of $\mathcal{H}$ of dimension $d$ and $\left\{\exp \left(-i \omega_{j} t\right)\right\}$ the corresponding eigenvalues. The evolution of an arbitrary initial density operator $\rho(0)$ over time $t$ is

$$
U(t) \rho(0) U^{\dagger}(t)=\sum_{j, k} e^{-i \omega_{j k} t} \rho_{j k}\left|\phi_{j}\right\rangle\left\langle\phi_{k}\right|
$$

for $\omega_{j k} \equiv \omega_{j}-\omega_{k}$ and $\rho_{j k} \equiv\left\langle\phi_{j}|\rho(0)| \phi_{k}\right\rangle$.

Entanglement for pure states $\left(\rho=\rho^{2}\right)$ with two subsystems is given by the entropy of the reduced density operator $\widetilde{\rho}(t)$ of either subsystem. The linear entropy $S=1-\operatorname{Tr}\left(\widetilde{\rho}^{2}\right)$ is a convenient measure of entanglement, with $S=0$ for no entanglement and $S=1-1 / d_{i}$ for maximum entanglement. The time-dependent entropy is

$$
S(t)=1-\sum_{j, k, l, m} C_{j k l m} e^{-i\left(\omega_{j k}+\omega_{l m}\right) t}
$$

with

$$
C_{j k l m}=\rho_{j k} \rho_{l m} \sum_{p, q, r, s}\left\langle u_{p}, v_{r} \mid \phi_{j}\right\rangle\left\langle\phi_{k} \mid u_{p}, v_{s}\right\rangle \times\left\langle u_{q}, v_{s} \mid \phi_{l}\right\rangle\left\langle\phi_{m} \mid u_{q}, v_{r}\right\rangle
$$

for $\left\{\left|u_{l}\right\rangle\right\},\left\{\left|v_{r}\right\rangle\right\}$ orthonormal bases for $\mathcal{H}^{(1)}$ and $\mathcal{H}^{(2)}$.

Diagonal elements $\rho_{i i}$ quantify support of $\rho$ on $U$ eigenstates $\left|\phi_{i}\right\rangle$. These eigenstates can be associated with regular and chaotic regimes $[15,16]$; hence a state $\rho$ can be identified with classical regular or chaotic regimes (or a combination) by its support on $U$ eigenstates. We employ this correspondence between support [10] and regular vs chaotic dynamics to characterize entanglement dynamics for an AMOL and other QC systems.

\section{ENTANGLEMENT DYNAMICS OF ATOMS IN A MAGNETO-OPTICAL LATTICE}

The AMOL system consists of atoms trapped in a onedimensional (1D) far off-resonance optical lattice created by two counterpropagating laser beams with an angle $\Theta_{L}$ between their linear polarizations. The light shift potential that results from the dipole interaction between the atom and the laser beam electric field $\boldsymbol{E}$ is [12]

$$
\hat{U}=-\frac{1}{4} \boldsymbol{E}^{*} \cdot \hat{\boldsymbol{\alpha}} \cdot \boldsymbol{E} .
$$

Here $\hat{\alpha}$ is the atomic polarizability tensor. For a multilevel atom, the polarizability tensor depends on the internal states of the atom and can be written as the sum of a scalar term, a vector term, and a tensor term.

In experiments using alkali-metal atoms [13], the total angular momentum is prepared in a hyperfine ground state with quantum number $F$. When the laser field is sufficiently detuned from resonance such that the excited state hyperfine splitting can be neglected, the light-shift potential as a function of the atomic position $z$ reduces to the sum of a scalar part (independent of the atom's spin state) and a vector part, which appears as an effective Zeeman interaction. The resultant Hamiltonian is [13]

$$
H=\frac{p^{2}}{2 m}+\frac{4}{3} V_{1} \cos \Theta_{\mathrm{L}} \cos 2 k z-\boldsymbol{\mu} \cdot \boldsymbol{B}_{\mathrm{eff}}(z)
$$

for

$$
\boldsymbol{\mu}=\hbar \gamma \boldsymbol{F}=-\mu_{\mathrm{B}} \boldsymbol{F} / F,
$$

$\gamma$ the gyromagnetic ratio, and $\boldsymbol{F}$ the total angular momentum vector of the hyperfine ground state, with the quantization axis along the $z$ direction. As an example, we consider ${ }^{133} \mathrm{Cs}$ with $F=4$ and $\mu_{\mathrm{B}} \boldsymbol{B}_{\text {eff }}(z)=-\frac{2}{3} V_{1} \sin \Theta_{\mathrm{L}} \sin 2 k z \boldsymbol{e}_{z}+B_{x} \boldsymbol{e}_{x}$, for $k$ the laser wave vector, $V_{1}$ the single-beam light shift, and $\boldsymbol{e}_{x}, \boldsymbol{e}_{z}$ unit vectors in the $x$ and $z$ directions. The $z$ component of $\boldsymbol{B}_{\text {eff }}(z)$ arises from the vector term in the atomic polarizability tensor while the $x$ component is due to an additional applied transverse magnetic field $B_{x}$, which is the tunable chaoticity parameter. The coupling between the spin precession and center-of-mass motion leads to entangled spinor wave packets. [In fact, Eq. (4) is applicable to more general systems if the periodic potential is replaced by a harmonic potential [17].]

In the classical limit, Eq. (3) describes a magnetic moment interacting with the same $\boldsymbol{B}_{\text {eff }}(z)$ [18], with $\boldsymbol{n}=\boldsymbol{F} / F$ the direction vector for the classical angular momentum. The classical four-dimensional phase space is parametrized by atomic position and momentum $(z, p)$ and direction $(\theta, \phi)$ of $\boldsymbol{F} / F$. This is equivalent to a system with two effective degrees of freedom. The resulting classical equations of motion are

$$
\frac{d z}{d t}=\frac{p}{m}
$$

$$
\begin{gathered}
\frac{d p}{d t}=-\frac{d}{d z}\left(\frac{4}{3} V_{1} \cos \Theta_{\mathrm{L}} \cos 2 k z+\mu_{B} \mathbf{n} \cdot \mathbf{B}_{\mathrm{eff}}(z)\right), \\
\frac{d \mathbf{n}}{d t}=\gamma\left[\mathbf{n} \times \mathbf{B}_{\mathrm{eff}}(z)\right] .
\end{gathered}
$$

Nonintegrability of these equations follows since there is only one constant of the motion, the energy.

We seek to study dynamical entanglement between spin and center-of-mass motion for lattice parameters that are accessible in current experiments. Therefore we choose $V_{1}$ $=160 E_{R}, \Theta_{L}=80^{\circ}$, and $\mu_{B} B_{x}=12 E_{R}$, for $E_{R}=\hbar^{2} k^{2} / 2 M$, the recoil energy. Classical Poincaré sections for these parameters and total energy $E=p^{2} / 2 M+V=-280 E_{R}$ reveal a mixed phase space with islands of regular motion embedded in the chaotic sea (Fig. 1). Quantum states are localized to phase space coordinates $(z, p, \theta, \phi)$ by preparation in a product of the motional and spin coherent states $|\alpha=z+i p\rangle|\theta, \phi\rangle$ [19].

An AMOL state localized around $(z, 0, \theta, \phi)$ can be prepared by cooling atoms to the ground state of the diabatic potentials. The lattice is then shifted until this state is centered at $z / \lambda$. The spin is rotated until the Bloch vector is pointing in the direction $(\theta, \phi)$. We pick an initial state that is 
(a)
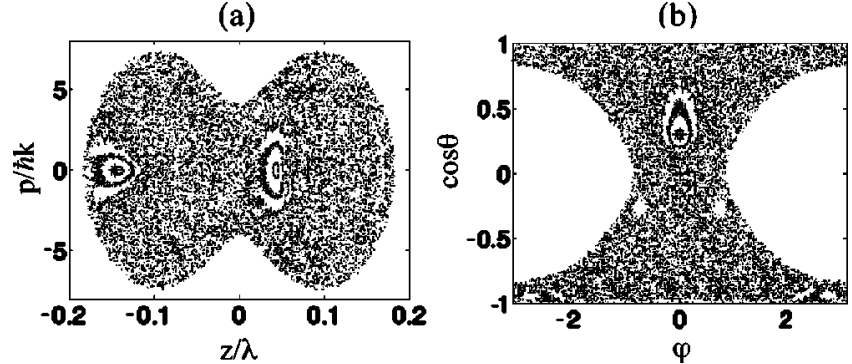

FIG. 1. Classical Poincaré sections for the AMOL at $E=$ $-280 E_{R}$ for $V_{1}=160 E_{R}, \Theta_{L}=80^{\circ}$, and $\mu_{B} B_{x}=12 E_{R}$ with (a) $\mu_{y}$ $=0, d \mu_{y} / d t>0$ and (b) $p=0, d p / d t>0$.

centered on an island in the Poincaré section in Fig. 1. For comparison we also consider an initial state in the chaotic sea.

The evolution of entanglement between spin and motional degrees of freedom, quantified by $S(t)$, for states that are initially regular or chaotic can be experimentally measured by reconstructing the time evolving reduced density matrix of the spin using tomographic techniques that have recently been demonstrated [14]. The populations in the different magnetic sublevels along $4 F+1$ different quantization axes can be experimentally measured using Stern-Gerlach measurements, and the $(2 F+1)^{2}$ elements of the spin density matrix can be calculated from these measurements. The linear entropy $S(t)$ of the spin can then be easily computed.

The predicted dynamical behavior of $S(t)$ in regular [Fig. 2(a)] versus chaotic [Fig. 2(b)] regions exhibits two main signatures of chaos. At short times, entanglement in the chaotic regime increases at a faster rate than for the regular regime, thereby supporting the concept that chaos can cause rapid generation of entanglement as predicted in other systems $[3,6]$. Also oscillations are prevalent for initially regular states but not for chaotic states (which has been also observed for the quantum kicked top [6]). We explain how the power spectrum of $S$ can provide a signature of chaos, as Lahiri suggested [7], by exploiting the $U$ eigenbasis.

$U$-eigenstate support for the states initially in the regular and chaotic regimes are depicted in Fig. 3. The initial state on the regular island has support dominated by four pairs of (a)

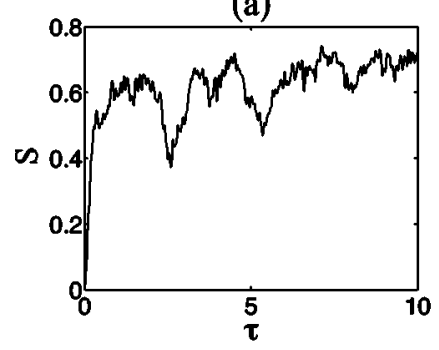

(b)

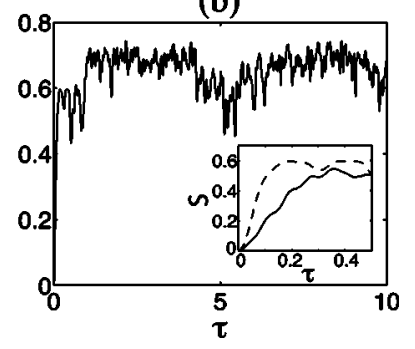

FIG. 2. Entanglement $S$ vs time $\tau=E_{R} t / \hbar$ in the AMOL for an initial state that is localized on (a) a regular island with $(z / \lambda, p / \hbar k, \theta, \phi)=(-0.15,0,1.27,0)$ and (b) the chaotic sea with $(z / \lambda, p / \hbar k, \theta, \phi)=(0.06,0, \pi / 2,0)$. The inset shows the initial increase of entanglement for the regular (solid) and chaotic (dashed) initial states. (a)

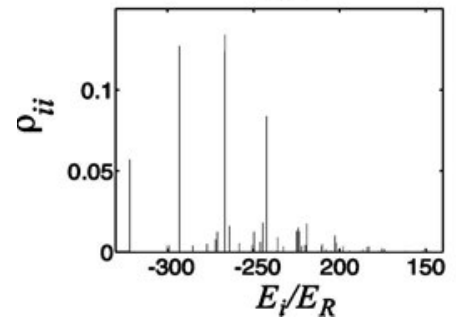

(b)

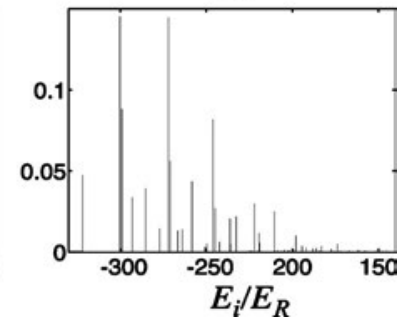

FIG. 3. Population $\rho_{i i}$ vs corresponding eigenenergy $E_{i}=\hbar \omega_{i}$ for the AMOL with an initial state localized in (a) a regular island with $(z / \lambda, p / \hbar k, \theta, \phi)=(-0.15,0,1.27,0)$ and (b) the chaotic sea with $(z / \lambda, p / \hbar k, \theta, \phi)=(0.06,0, \pi / 2,0)$.

regular eigenstates with each pair nearly degenerate. This support over few eigenstates is responsible for the quasiperiodic evolution of linear entropy, and Fig. 4(a) shows that excellent replication of entanglement dynamics is possible by only including these four pairs of eigenstates. In contrast, the initial state in the chaotic sea has support over a larger number of the chaotic set of eigenstates extending over a broader frequency spectrum [Fig. 3(b)], due to a breakdown of semiclassical theory in the chaotic regime $[15,16]$.

A rigorous understanding of entanglement evolution emerges by noting that $S(t)$ in Eq. (3) depends on eigenfrequency-difference sums $\omega_{i j}+\omega_{k l}$, which can be identified in the power spectrum of $S(t)$. The fast oscillations in Fig. 2(a) are due to the large differences $\delta \omega$ between the four main peaks in Fig. 3(a), and small frequency differences between almost degenerate eigenstate pairs at each peak in Fig. 3(a) result in slow oscillations with long periods as seen in the long-term behavior of the entanglement in Fig. 4(b). The terms $\omega_{i j}+\omega_{k l}$ that appear in the evolution correspond not just to differences in the eigenfrequencies, but also can be a sum of $\omega_{i j}+\omega_{k l}$. For example, the main oscillation in Fig. 2(a) is due to the sum of two difference frequencies. These frequencies can be extracted from a Fourier transform of the dynamics, which reveals a discrete power spectrum in contrast to the more continuous spectrum for the initially chaotic state.

The key feature of dynamical entanglement for our purpose is the initial increase of entanglement for a chaotic state, which is more rapid than for the regular state (see inset
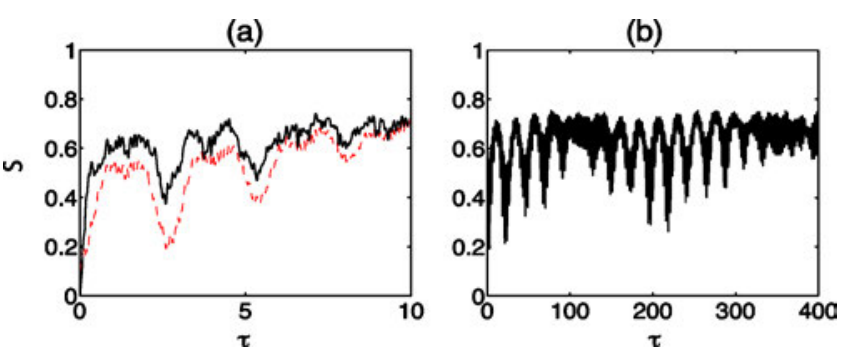

FIG. 4. (a) For the initial state localized on the regular island the entanglement dynamics (solid curve) can be reproduced by only considering the evolution of the four main pairs of eigenstates (dashed curve). The long term behavior (b) shows quasiperiodic motion with multiple frequencies. 
of Fig. 2). This rapid rise in entanglement is not unique to the AMOL system $[3,6]$. The rate of increase of entanglement is obtained by expanding Eq. (1) at $t=0$, which reveals a quadratic increase as a function of time, $S=\left(t / t_{0}\right)^{2}$ with $t_{0}$ $=0.01$; this behavior is surprising at first because an exponential increase is expected for states in the chaotic regime and a quadratic increase for states in the regular regime [8]. The predicted quadratic versus exponential behavior is obtained by relating the purity of the reduced density matrix to a classical time correlator [8]. Of course this expectation applies for the asymptotic semiclassical regime, but our system lies in a quantum regime, hence the chaotic state is not well localized, with resultant non-negligible support over the regular regime yielding a quadratic increase in entanglement. For the initial state in the chaotic regime,

$$
\Delta x / \lambda \approx 0.07, \quad \Delta p / \hbar k \approx 2.7,
$$

and

$$
\Delta \mu_{x}=0, \quad \Delta \mu_{z}=\Delta \mu_{y}=1 / \sqrt{2} .
$$

\section{ENTANGLEMENT DYNAMICS IN A QUANTUM KICKED TOP}

Our methods to analyze the AMOL are generic and generalize to other unitarily evolving QC systems as we now show for the quantum kicked top with Hamiltonian [9-11]

$$
H=\frac{\kappa}{2 j \tau} J_{z}^{2}+p J_{y} \sum_{n=-\infty}^{\infty} \delta(t-n \tau)
$$

for $J_{x}, J_{y}, J_{z} \operatorname{su}(2)$ operators and $\kappa$ the chaoticity parameter. The QKT can be constructed from a collection of $N=2 j$ qubits in the symmetric representation with collective spin operators

$$
J_{\alpha}=\sum_{i=1}^{N} \frac{\sigma_{i \alpha}}{2},
$$

and $\left\{\sigma_{i \alpha}\right\}$ the Pauli operators for the ith qubit [6]. For $\kappa=3$, $\tau=1, p=\pi / 2$ entanglement behavior is similar to that of the trapped atoms described here [6]. Bipartite entanglement between a pair of qubits and the remaining qubits reveals quasiperiodic evolution for an initial state centered on an elliptic fixed point. For a state centered in the chaotic sea, no quasiperiodic motion is present, and just as in our AMOL, an initial rapid increase of entanglement is observed confirming this generic behavior.

Support of an initial state over $\left(U=F^{n}\right)$ eigenstates, of the Floquet operator

$$
F=\exp \left(-i \kappa J_{z}^{2} / 2 j \tau\right) \exp \left(-i p J_{y}\right)
$$

is shown for an initial state centered on the elliptic fixed point and one in the chaotic region of Ref. [6] (Fig. 5). The initial state centered in the regular region can be mainly decomposed into a few (size $\sqrt{N}$ ) "regular" eigenstates [10]. In this case the state that we have localized at a fixed point has most of its support on three eigenstates, of which two are (a)

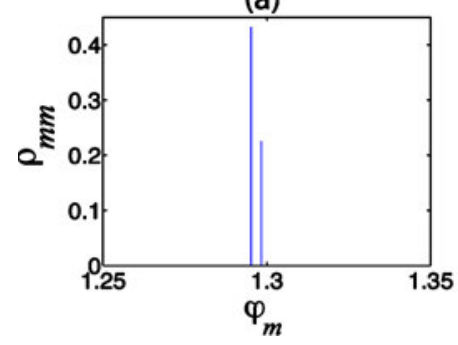

(b)

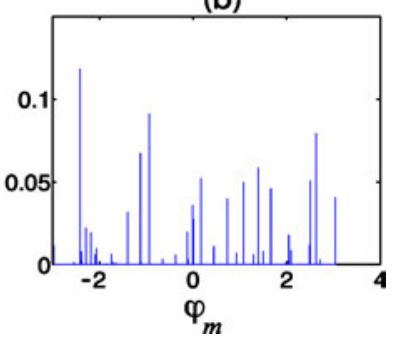

FIG. 5. (Color online) Population $\rho_{m m}$ vs corresponding eigenphases $\phi_{m}$ for the QKT with an initial state localized in (a) a regular island and (b) the chaotic sea in Fig. 1 of [6].

degenerate. The difference between the corresponding eigenphases $\phi_{m}, \delta \phi=0.003$, determines the frequencies of oscillation in the evolution of $S$ [Fig. 6(a)].

The flat entanglement power spectrum for the chaotic state is due to the broad support (size $N$ ) [10] of the initial state on the "chaotic" $U$ eigenstates [Fig. 5(b)]. Because $N$ $=50$ qubits is in the semiclassical regime, the distinction between regular and chaotic entanglement dynamics is more pronounced than what we observed for the AMOL, which was not as semiclassical. (As we consider ${ }^{133} \mathrm{Cs}$ for the AMOL, $F=4$ is fixed and thus not adjustable to reach the semiclassical regime.) Moreover the rise time for the initial chaotic state is exponential [8] which can be regarded as a signature of quantum chaos.

The QKT system of $N$ qubits behaves collectively like a system with one degree of freedom unlike the AMOL which has two degrees of freedom: spin and motion. Coupling between two QKTs, however, allows for entanglement dynamics between two coupled degrees of freedom to be observed, but entanglement is suppressed in the strong chaotic regime [5]. Our results for the single QKT provide an intuitive understanding of this suppression of entanglement. Since chaos enhances the entanglement between the qubits in each kicked top, the qubits cannot also be highly entangled with the qubits of the other top.

\section{CONCLUSION}

In conclusion, we have presented entanglement dynamics for an experimentally feasible QC system of atoms trapped

(a)

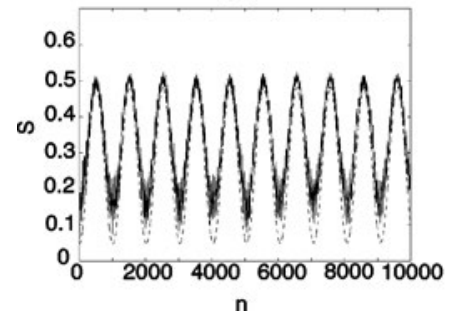

FIG. 6. (a) For the regular initial state of the QKT the entanglement dynamics (solid curve) can be reproduced by considering the evolution of the three highest weighted eigenstates (dashed curve) in Fig. 5(a). (b) The rise time for the state in the chaotic sea is roughly exponential. 
in a magneto-optical lattice. For realistic experimental parameters, quantum signatures of chaos exist in the dynamics of entanglement, specifically in the initial rise and the power spectrum, even when the system is not in a semiclassical regime, and there is a rapid increase of entanglement for initial states in the chaotic regime. Our results show that the AMOL system is a convenient setting in which entanglement dynamics can be experimentally observed.

Our analysis relies on studying support over the unitary evolution eigenbasis, which applies generically to other unitarily evolving QC systems, as we demonstrate with the quantum kicked top. This provides a simple method for predicting the behavior of a system by only considering the initial state and the eigenstates of the system. Thus we have introduced a means for extending QC experiments to more than one degree of freedom, observing and understanding entanglement dynamics in such systems, exploiting quantum chaos for a rapid increase in entanglement, and explaining when chaos enhances vs diminishes entanglement generation for initially chaotic states.

\section{ACKNOWLEGMENTS}

We appreciate valuable discussions with I. Deutsch, P. Jacquod, and Xiaoguang Wang (who also provided computer codes for obtaining data in Fig. 6). Numerical results for the AMOL system were partially based on codes developed by $\mathrm{J}$. Grondalski. This project has been supported by an Australian Research Council Large Grant and Alberta's Informatics Circle of Research Excellence (iCORE) and the National Science Foundation under Grant No. PHY-009569.
[1] F. Haake, Quantum Signature of Chaos (Springer-Verlag, Berlin, 1991); H.-J. Stockmann, Quantum Chaos: An Introduction (Cambridge University Press, Cambridge, U.K., 1999); C. Zhang, J. Liu, M. G. Raizen, and Q. Niu, Phys. Rev. Lett. 92, 054101 (2004); D. A. Steck, W. H. Oskay, and M. G. Raizen, Science 293, 274 (2001); B. G. Klappauf, W. H. Oskay, D. A. Steck, and M. G. Raizen, Physica D 131, 78 (1999).

[2] M. A. Nielsen and I. L. Chuang, Quantum Computation and Quantum Information (Cambridge University Press, Cambridge, U.K., 2000).

[3] K. Furuya, M. C. Nemes, and G. Q. Pellegrino, Phys. Rev. Lett. 80, 5524 (1998); P. A. Miller and S. Sarkar, Phys. Rev. E 60, 1542 (1999).

[4] A. Lakshminarayan, Phys. Rev. E 64, 036207 (2001); J. N. Bandyopadhyay and A. Lakshminarayan, Phys. Rev. Lett. 89, 060402 (2002); S. Bettelli and D. L. Shepelyansky, Phys. Rev. A 67, 054303 (2003); A. J. Scott and C. M. Caves, J. Phys. A 36, 9553 (2003).

[5] H. Fujisaki, T. Miyadera, and A. Tanaka, Phys. Rev. E 67, 066201 (2003); M. Znidaric and T. Prosen, J. Phys. A 36, 2463 (2002); T. Prosen and T. Seligman, ibid. 35, 4707 (2002).

[6] X. G. Wang, S. Ghose, B. C. Sanders, and B. Hu, Phys. Rev. E 70, 016217 (2004).
[7] A. Lahiri, e-print quant-ph/0302029.

[8] P. Jacquod, Phys. Rev. Lett. 92, 150403 (2004).

[9] H. Frahm and H. J. Mikeska, Z. Phys. B: Condens. Matter 60, 117 (1985).

[10] F. Haake, M. Kus, and R. Scharf, Z. Phys. B: Condens. Matter 65, 381 (1987).

[11] B. C. Sanders and G. J. Milburn, Z. Phys. B: Condens. Matter 77, 497 (1989).

[12] P. S. Jessen and I. H. Deutsch, Adv. At., Mol., Opt. Phys. 37, 95 (1996).

[13] I. H. Deutsch and P. S. Jessen, Phys. Rev. A 57, 1972 (1998); I. H. Deutsch et al., J. Opt. B: Quantum Semiclassical Opt. 2, 633 (2000).

[14] G. Klose, G. Smith, and P. S. Jessen, Phys. Rev. Lett. 86, 4721 (2001).

[15] I. C. Percival, J. Phys. B 6, L229 (1973).

[16] A. Einstein, Verh. Dtsch. Phys. Ges. 19, 82 (1917).

[17] A. J. Leggett et al., Rev. Mod. Phys. 59, 1 (1987); A. J. Leggett et al., ibid. 67, 725 (1995); E. T. Jaynes and F. W. Cummings, Proc. IEEE 51, 89 (1963).

[18] S. Ghose, P. M. Alsing, and I. H. Deutsch, Phys. Rev. E 64, 056119 (2001).

[19] F. T. Arrechi et al., Phys. Rev. A 6, 2211 (1972). 